\title{
12
}

\section{AC Electrokinetic Stirring and Focusing of Nanoparticles}

\author{
Marin Sigurdson, Dong-Eui Chang, Idan Tuval, \\ Igor Mezic, and Carl Meinhart \\ Department of Mechanical Engineering, University of California-Santa Barbara
}

\subsection{INTRODUCTION}

Immunoassay-based sensors rely on specific antigen-antibody binding for identification of proteins. These sensors have applications in both clinical laboratories for medical diagnostics, and in research laboratories for highly-multiplexed testing. In these cases, throughput is a key consideration. One factor limiting test duration is diffusion of analyte to the reporter. An incubation step of minutes to hours is required for diffusion-limited reactions to reach detectable levels. These tests are usually performed at centralized labs where high throughput is achieved through robotics and highly parallel assays. However, if the assay could be moved from a centralized lab to the point of care, the test could be much faster, as well as smaller, while maintaining high sensitivity.

In response to this need, microfluidic assays for diagnostics have developed dramatically in recent years. This facilitates the use of the lab-on-a-chip concepts for point-of-care diagnosis, and high throughput screening for molecular diagnostics. The small length scales associated with microfluidic devices permit small sample sizes and shorter assay incubation times. In addition, on-chip sample preparation reduces fluid handling steps. Though greatly aided by their small length scales, these assays can still be diffusion limited. Ac electrokinetic stirring can potentially reduce incubation times, and can be adaptable to a wide variety of assay configurations. 


\subsection{AC ELECTROKINETIC PHENOMENA}

Ac electrokinetics refers to induced particle or fluid motion resulting from externally applied ac electric fields. Dc electrokinetics has been widely successful for lab-on-a-chip applications such as capillary zone electrophoresis (Aclara and Caliper [1, 5], capillary gel electrophoresis for DNA fractionation [19] and electroosmotic pumping [3, 4]. However, ac electrokinetics has received relatively little attention. Ac electrokinetics have the advantages over its dc counterpart by (1) largely avoiding electrolysis, and (2) operating at relatively lower voltages $(1 \sim 20 \mathrm{~V})$. Ac electrokinetics can be classified into three broad areas: dielectrophoresis (DEP), electrothermal flow, and AC electro-osmosis [18].

Dielectrophoresis is a force arising from differences in polarizability between the particle and the fluid medium in the presence of a non-uniform electric field. DEP has been used to separate blood cells and to capture DNA molecules [7, 12, 21, 23, 24], provides an overview). However, since the force scales with the cube of particle radius, it has limited effectiveness for manipulating nanoscale molecules (such as $10 \mathrm{~nm}$-scale antigen).

AC Electroosmosis arises when the tangential component of the electric field interacts with a field-induced double layer along a surface. It becomes less important for sufficiently large electric field frequencies. For example, in an aqueous saline solution with an electrical conductivity of $\sigma=2 \times 10^{-3} \mathrm{~S} / \mathrm{m}$, it is predicted that AC electroosmosis is not important above $100 \mathrm{kHz}$ [17].

Transport enhancement for small proteins may be most successful through electrothermally driven flow (ETF). A non-uniform electric field produces non-uniform Joule heating of the fluid, which gives rise to spatial variations in electrical conductivity and permittivity. These variations create electrical charge density variations, even for electrically neutral fluids. The electrical charge density coupled with the applied electric field gives rise to Coulomb body forces in the fluid. The Coulomb body forces induce local fluid stirring. These characteristic swirling flow patterns can be used to transport suspended molecules towards a heterogeneous binding region, or for non-local focusing of particles away from the electrode surface. This can increase the binding rate of immuno-assays, and therefore can improve the response time and overall sensitivity of microfluidic-based sensors.

\subsection{DEP: A SYSTEM THEORY APPROACH}

If a dielectric particle is suspended in an ac electric field, acting within a dielectric medium, it will polarize. The magnitude and direction of the induced dipole will depend on the frequency and the magnitude of the applied electric field and the dielectric properties of the particle and the medium. A nonhomogeneous electric field acting on the induced dipole in turn produces a force on the dipole, called the dielectrophoretic (DEP) force. Thus, dielectrophoresis is the force exerted on a particle in the presence of a non-uniform electric field [16] (see Fig. 12.1).

To explain this in more detail we describe a systems theory of dielectrophoresis, as developed in Chang et al. 2003. The induced dipole moment, $\boldsymbol{m}(q, t)$, in a particle due to an external electric field, $\boldsymbol{E}(q, t)$, depends linearly on the electric field $[6,10]$. This linear 

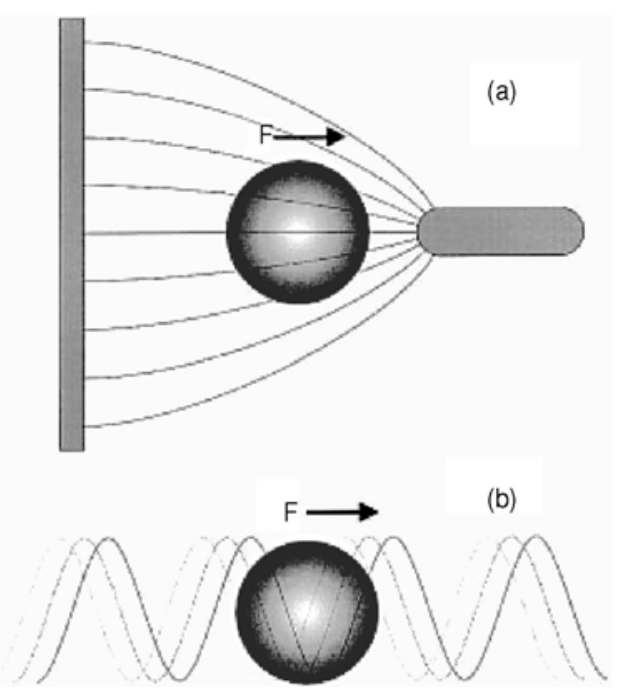

FIGURE 12.1. Particles suspended in a nonhomogeneous ac electric field experience a force due to the interaction of the induced dipole moment and the applied electric field. In a) force due to magnitude gradient is represented. In b) force due to phase gradient of the electric field is shown (figure from [9]).

relation can be written as

$$
\hat{\mathbf{m}}(q, s)=G(s) \hat{\mathbf{E}}(q, s)
$$

where $\hat{\mathbf{m}}(q, s), \hat{\mathbf{E}}(q, s)$ are the Laplace transforms of $\mathbf{m}(q, t), \mathbf{E}(q, t)$, respectively, and $G(s)$ is the transfer function. When a spherical particle with the permittivity $\varepsilon_{p}$, the conductivity $\sigma_{p}$ and radius $r$, lies in a medium with the permittivity $\varepsilon_{m}$ and the conductivity $\sigma_{m}$, the transfer function $G(s)$ is given by

$$
G(s)=4 \pi r^{3} \epsilon_{m} \frac{\left(\epsilon_{p}+\frac{\sigma_{p}}{s}\right)-\left(\epsilon_{m}+\frac{\sigma_{m}}{s}\right)}{\left(\epsilon_{p}+\frac{\sigma_{p}}{s}\right)+2\left(\epsilon_{m}+\frac{\sigma_{m}}{s}\right)},
$$

where $G(s) /\left(4 \pi r^{3} \varepsilon_{\mathrm{m}}\right)$ is the so-called Clausius-Mossotti function [10, 78]. Notice that the transfer function depends on the electric properties both of the particle and of the medium. The dielectrophoretic force, $\boldsymbol{F}_{d e p}$, on the particle due to the interaction between the induced dipole and the electric field, is given by

$$
\mathbf{F}_{\mathrm{dep}}(q, t)=(m(q, t) \cdot \nabla) \mathbf{E}(q, t),
$$

The time-averaged force, $\left\langle\boldsymbol{F}_{d e p}\right\rangle$, is defined by

$$
\left\langle\mathbf{F}_{\mathrm{dep}}\right\rangle(q)=\lim _{T \rightarrow \infty} \frac{1}{T} \int_{0}^{T} \mathbf{F}_{\mathrm{dep}}(q, t) d t
$$


assuming this limit exists. These equations give the relationship between the electric field and the resultant dielectrophoretic force on particles.

We illustrate this formalism with the computation of dielectrophoretic forces corresponding to various (curl-free) electric fields; similar computations can be used to compute DEP forces of various geometries and time-dependencies. We will consider the following four cases:

\section{Case 1. The electric field is:}

$$
\mathbf{E}(q, t)=\mathbf{E}_{1}(q) \cos (\omega t)
$$

Giving:

$$
\begin{aligned}
\mathbf{m}(q, t) & =|G(j \omega)| \cos (\omega t+\measuredangle G(j \omega)) \mathbf{E}_{1}(q), \\
\mathbf{F}_{\mathrm{dep}}(q, t) & =\frac{1}{2}|G(j \omega)| \cos (\omega t+\measuredangle G(j \omega)) \cos (\omega t) \nabla\left|\mathbf{E}_{1}(q)\right|^{2}, \\
\left\langle\mathbf{F}_{\mathrm{dep}}\right\rangle(q) & =\frac{1}{4} \operatorname{Re}[G(j \omega)] \nabla\left|\mathbf{E}_{1}(q)\right|^{2} .
\end{aligned}
$$

Notice that $\left\langle\boldsymbol{F}_{\text {dep }}\right\rangle$ moves particles toward the maxima of the magnitude of the electric field if $\operatorname{Re}[\mathrm{G}(\mathrm{j} \omega)]>0$, see Fig. 12.1. The maxima of the magnitude of electric fields usually occur at the edge of electrodes. This is known as positive DEP or p-DEP. Negative DEP occurs when the DEP force is away from intense electric fields, and is denoted by n-DEP.

Case 2. The electric field is periodic with period $T>0$ as:

$$
\mathbf{E}(q, t)=\mathbf{E}(q, t+T)
$$

Here, we can express the electric field as a Fourier series:

$$
\mathbf{E}(q, t)=\mathrm{E}_{0}(q)+\sum_{n=1}^{\infty}\left(\mathbf{E}_{n}^{c}(q) \cos (n \omega t)+\mathbf{E}_{n}^{s}(q) \sin (n \omega t)\right) .
$$

Then,

$$
\begin{aligned}
\left\langle\mathbf{F}_{\mathrm{dep}}\right\rangle(q)= & \frac{1}{2} G(0) \nabla\left|\mathrm{E}_{0}\right|^{2}+\sum_{n=1}^{\infty} \frac{1}{2} \operatorname{Re}[G(j n \omega)] \nabla\left(\left|\mathrm{E}_{n}^{c}\right|^{2}+\left|\mathrm{E}_{n}^{s}\right|^{2}\right) \\
& +\sum_{n=2}^{\infty} \frac{1}{2} \operatorname{Im}[G(j n \omega)] \nabla \times\left(\mathrm{E}_{n}^{c} \times \mathbf{E}_{n}^{s}\right) .
\end{aligned}
$$

Writing the periodic field in the following form

$$
\mathbf{E}(q, t)=\left[\begin{array}{l}
E_{0, x}(q) \\
E_{0, y}(q) \\
E_{0, z}(q)
\end{array}\right]+\sum_{n=1}^{\infty}\left[\begin{array}{l}
E_{n, x}(q) \cos \left(n \omega t+\phi_{n, x}(q)\right) \\
E_{n, y}(q) \cos \left(n \omega t+\phi_{n, y}(q)\right) \\
E_{n, z}(q) \cos \left(n \omega t+\phi_{n, z}(q)\right)
\end{array}\right],
$$

we obtain the following form of the averaged dielectrophoretic force:

$$
\begin{aligned}
\left\langle\mathbf{F}_{\mathrm{dep}}\right\rangle(q)= & \frac{1}{2} G(0) \nabla\left|\mathbf{E}_{0}\right|^{2}+\sum_{n=1}^{\infty} \frac{1}{4} \operatorname{Re}[G(j n \omega)] \nabla\left(E_{n, x}^{2}+E_{n, y}^{2}+E_{n, z}^{2}\right) \\
& +\sum_{n=1}^{\infty} \frac{1}{2} \operatorname{Im}[G(j n \omega)]\left(E_{n, x}^{2} \nabla \phi_{n, x}+E_{n, y}^{2} \nabla \phi_{n, y}+E_{n, z}^{2} \nabla \phi_{n, z}\right) .
\end{aligned}
$$


In dielectrophoresis literature, the time-dependence of the force is typically sinusoidal. However, it is sometimes convenient to use non-sinusoidal periodic signals such as square waves, saw-tooth waves, to achieve a desired effect. The formulas above allow us to compute the corresponding time-averaged force. Notice that the electric field is not only periodic but also traveling. In addition, the dielectrophoretic force depends on the imaginary part of the transfer function and the gradient of the phases. This results in traveling wave DEP, or tw-DEP, illustrated in Fig. 12.1b.

\section{Case 3. An almost-periodic electric field of the form:}

$$
\mathbf{E}(q, t)=\mathbf{E}_{0}(q)+\sum_{n=1}^{\infty}\left(\mathbf{E}_{n}^{c}(q) \cos \left(\omega_{n} t\right)+\mathbf{E}_{n}^{s}(q) \sin \left(\omega_{n} t\right)\right)
$$

where all the nonzero $\omega_{\mathrm{n}}$ are distinct. The averaged dielectric force is given by

$$
\begin{aligned}
\left\langle\mathrm{F}_{\mathrm{dep}}\right\rangle(q)= & \frac{1}{2} G(0) \nabla\left|\mathbf{E}_{0}\right|^{2}+\sum_{n=1}^{\infty} \frac{1}{4} \operatorname{Re}\left[G\left(j \omega_{n}\right)\right] \nabla\left(\left|\mathbf{E}_{n}^{c}\right|^{2}\left|\mathrm{E}_{n}^{s}\right|^{2}\right) \\
& +\sum_{n=1}^{\infty} \frac{1}{2} \operatorname{Im}\left[G\left(j \omega_{n}\right)\right] \nabla \times\left(\mathbf{E}_{n}^{c} \times \mathbf{E}_{n}^{c}\right) .
\end{aligned}
$$

\section{Case 4. A general time-varying electric field $\mathrm{E}(q, t)$ :}

The corresponding dielectric force can be written in a compact form as follows:

$$
\mathbf{F}_{\mathbf{d e p}}(q, t)=\int_{0}^{t} g(t-\tau)(\mathbf{E}(q, \tau) \cdot \nabla) \mathbf{E}(q, t) d \tau
$$

where $g(t)$ is the impulse response of the dipole system, $G(s)$.

\subsection{NON-LOCAL DEP TRAPPING}

The above theory is valid when the fluid flow is negligible. However, if ac electrokinetically-induce fluid flow (such as electrothermal or ac electroosmotic flow) is present, it can induce both desirable and undesirable effects. In the case of n-DEP, particles can be trapped close to the electrodes, instead of being induced away from the electrodes. In the case of p-DEP, it may not be desirable for particles to collect at the electrodes.

By utilizing carefully the effects of electrokinetically-induced fluid motion, one can focus particles at a non-local region away from the electrode surfaces using p-DEP, leading to orders of magnitude increase in local concentration of particles. Here, we discuss the theory behind this focusing phenomenon, based on the work in [20].

As described in the previous sections, an electric field can induce fluid motion through an electrothermal force. Experimental evidence, as well as full numerical simulations, show convective rolls centered at the electrode edges [11, 18, 22]. The fluid velocity ranges from $1-100 \mu \mathrm{m} / \mathrm{s}$, with an exponential decay as we move away from the electrodes. The boundary conditions are: no-slip at the bottom of the device, and both the horizontal component of the velocity and the normal derivative of the vertical velocity are zero at the symmetry planes. 


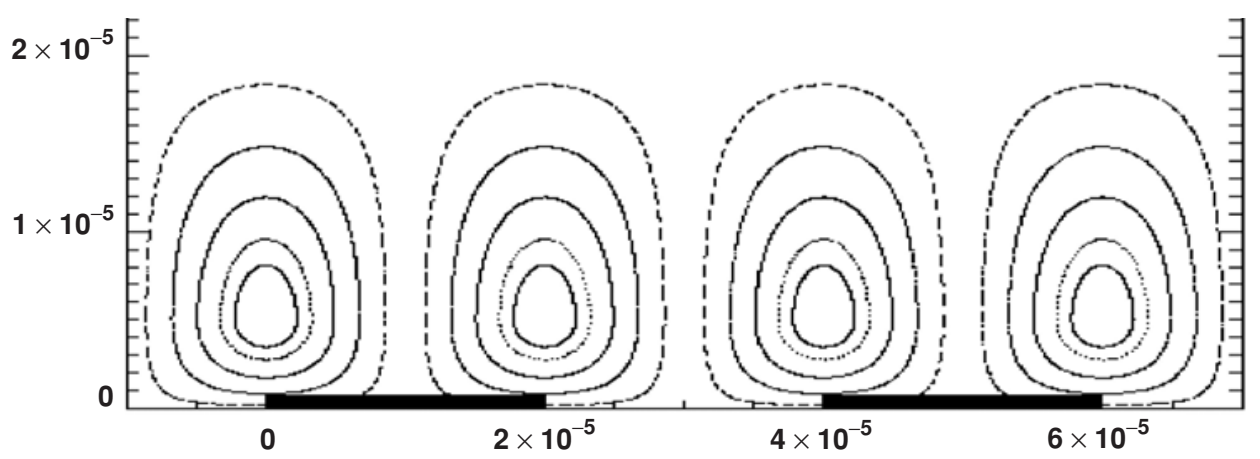

FIGURE 12.2. Streamlines of the cellular flow used in the model.

A simple model that captures these ideas was described in [20]. For an interdigitated array of electrodes a cellular flow is produced, and is depicted in Fig. 12.2.

One possible stream function is

$$
\psi_{\text {steady }}=\mathbf{u}_{\mathbf{0}} \cdot \mathbf{y}^{2} \mathbf{e}^{-\mathbf{y} / \beta} \cos (\pi \mathbf{x}),
$$

where the flow velocity naturally satisfies the incompressibility condition. The parameter $\beta$ determines the position of the center of the rolls.

Inertia in micron-size devices can be neglected, and the velocity of the particles can be obtained directly from the DEP force, buoyancy, drag force and Brownian motion, and can be described by the following stochastic ordinary differential equation.

$$
d q=\left(u(q)+\frac{\left\langle\mathbf{F}_{d e p}\right\rangle(q)}{6 \pi \eta r}+\left(\rho_{p}-\rho_{f}\right) \cdot \frac{2 r^{2}}{9 \eta} \cdot g\right) d t+d W_{t}
$$

where $u(q)$ is the fluid velocity at $q$ and $W_{t}$ is the Brownian motion of variance $2 D=$ $k T / 3 \pi \eta r$, where $k$ is the Boltzmann constant, $\eta$ is the viscosity of the fluid and $T$ is the temperature. The relative importance of the first three (deterministic force) terms, given the particle and fluid physical properties, depends basically on three parameters: the applied voltage $V$, the radius of the particle $r$, and the size of the electrode device $d$. The relative influence of fluid flow and Brownian motion gets progressively larger for smaller particles, and the buoyancy term becomes important only far from the electrodes where both the flow and DEP force are small.

For particles larger than a few microns, Brownian motion becomes less important and under certain circumstanced may be neglected [20]. The discussion in Tuval et al. [20] is in the context of dynamical systems methods. Two effects of different nature must be noted. Far from the electrodes, where the fluid velocity is smaller, the flow acts only as a small perturbation of the no-flow state. Therefore, the fixed points that exist due to the balance between negative DEP force and positive buoyancy, persist under the perturbation. The basic change is the accumulation of most of the particles in a small trapping area above the electrodes. This main effect has been pointed in several experiments [8, 13]. Under positive DEP, particles tend to accumulate at the edges of the electrodes. But fluid flow, that 


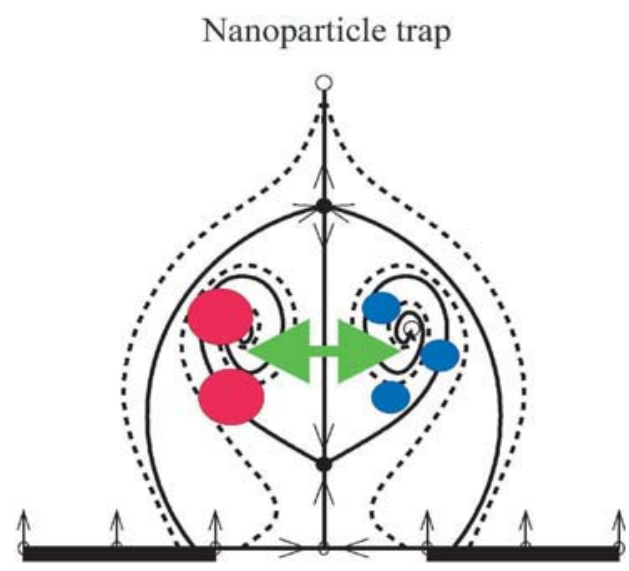

FIGURE 12.3. Sketch of the dynamical behavior of particles in the trapping zone.

is also stronger in that region, can influence the dynamics and move the particles across the electrode surface, finally collecting them above the center of the electrodes $[15,18]$.

A second effect that takes place close to the electrode surfaces is the creation of a closed zone from which particles can not escape There are two qualitatively different behaviors; some particles are trapped in a closed area above the electrodes or in the gap between electrodes depending on the sense of rotation of the flow, whereas others escape from the flow influence under negative DEP. A sketch of its dynamical structure of the trapping zone is depicted in Fig. 12.3.

Particles in the trapping zone are attracted towards two foci. Particles outside the trapping zone escape from the flow influence and finally reach and equilibrium position due to positive buoyancy, as in the absence of flow. One problem of interest that can be addressed using control theory methods is stabilization of the trapping zone.

\subsection{ELECTROTHERMAL STIRRING}

The finite element simulation software Femlab (Comsol; Stokholm, Sweden) is used for analysis of electrothermally-induced flow and subsequent enhanced binding in the cavity. First, the two-dimensional quasi-static potential field for two electrodes along the cavity wall is calculated, according to Laplace's Equation, $\nabla^{2} \mathbf{V}=0$. The resulting base electric field, given by $\vec{E}=-\nabla V$ gives rise to a non-uniform temperature field through Joule heating. Ignoring unsteady effects and convection (low Peclet number), and balancing thermal diffusion with Joule heating yields

$$
k \nabla^{2} T+\sigma E^{2}=0
$$

where $T$ is temperature, $E$ is the magnitude of the electric field, and $k$ and $\sigma$ are the thermal and electrical conductivities. Thermal boundary conditions are insulating on the channel surfaces. The metal electrodes are isothermal. The treatment of the electrodes 
(a) Temperature rise (K)
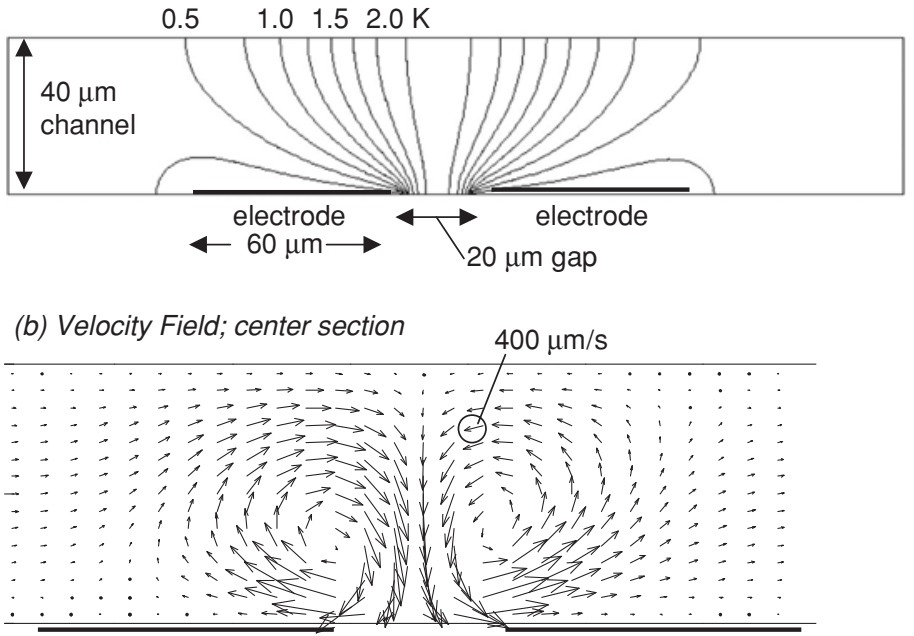

FIGURE 12.4. Simulation of electrothermally-driven flow in a $40 \mu \mathrm{m}$ channel using Femlab software. (a) Nonuniform temperature distribution created by Joule heating, and (b) Electrothermally-driven fluid motion. The pressure driven channel flow is moving from left to right at an average velocity of $100 \mu \mathrm{m} / \mathrm{s}$. The velocity of the electrothermally-driven flow is of order $400 \mu \mathrm{m} / \mathrm{s}$ and is characterized by a pair of counter rotating vortices.

as isothermal is appropriate for electrodes of sufficient thickness relative to length. The resulting temperature field is shown in Fig. 12.4a.

Gradients in temperature produce gradients in permittivity and conductivity in the fluid. For water $(1 / \sigma)(\partial \sigma / \partial T)=+2 \%$ nd $(1 / \varepsilon)(\partial \varepsilon / \partial T)=-0.4$ per degree Kelvin. These variations in electric properties produce gradients in charge density and perturb the electric field. Assuming the perturbed electric field is much smaller than the applied electric field, and that advection of electric charge is small compared to conduction, the time-averaged electrothermal force per unit volume for a non-dispersive fluid can be written as [18]

$$
\vec{F}_{E T}=-0.5\left[\left(\frac{\nabla \sigma}{\sigma}-\frac{\nabla \varepsilon}{\varepsilon}\right) \vec{E}_{r m s} \frac{\varepsilon \vec{E}_{r m s}}{1+(\omega \tau)^{2}}+0.5\left|\vec{E}_{r m s}\right|^{2} \nabla \varepsilon\right]
$$

where $\tau=\varepsilon / \sigma$ is the charge relaxation time of the fluid medium and the incremental temperature-dependent changes are

$$
\nabla \varepsilon=\left(\frac{\partial \varepsilon}{\partial T}\right) \nabla T, \quad \nabla \sigma=\left(\frac{\partial \sigma}{\partial T}\right) \nabla T
$$

The first term on the right hand side of Eq. (12.18) is the Coulomb force, and is dominant at low frequencies. The second term is the dielectric force, and is dominant at high frequencies. The crossover frequency scales inversely with the charge relaxation time of the fluid; an aqueous solution with conductivity $10^{-2} \mathrm{~S} / \mathrm{m}$ has a crossover frequency around $14 \mathrm{MHz}$. 
The electrothermal force shown in Eq. (12.18) is a body force on the fluid. The motion of the fluid can determined by solving the Stokes' equation for zero Reynolds number fluid flow, such that

$$
0=-\nabla \rho+\mu \nabla^{2} \vec{u}+\vec{F}_{E T}
$$

where $\vec{u}$ is the fluid velocity, $p$ is the pressure in the fluid, and $\mu$ is the dynamic viscosity of the fluid. Figure $12.4 \mathrm{~b}$ shows the resulting velocity field. The velocity of the ETF is of order $400 \mu \mathrm{m} / \mathrm{s}$, and characterized by a pair of counter rotating vortices, which may circulate the fluid effectively. The velocity field is similar to the streamlines shown in Fig. 12.2.

\subsection{ENHANCEMENT OF HETEROGENEOUS REACTIONS}

The effect of electrothermally-driven motion upon heterogeneous binding rates is examined in the following section. The convective scalar equation is solved to predict the suspended concentration $C(x, y)$ of antigen within the microchannel:

$$
\frac{\partial C}{\partial t}+\vec{u} \cdot \nabla C=D \nabla^{2} C
$$

where $\vec{u}$ is the fluid velocity and $D=2 \times 10^{-11} \mathrm{~m}^{2} \mathrm{~s}^{-1}$ is the diffusivity of an antigen. An antigen concentration of $C_{0}=0.1 \mathrm{nM}$ is introduced into the left hand side of the channel, for time $t>0$. Since the base flow is parabolic, analyte will be transported downstream most rapidly at the channel center (see Fig. 12.5a). After 1 second, the highest analyte concentration extends into the center of the channel, but no analyte concentration has yet reached the sensor binding site. When an alternating electrical potential of $7 \mathrm{Vrms}$ is applied

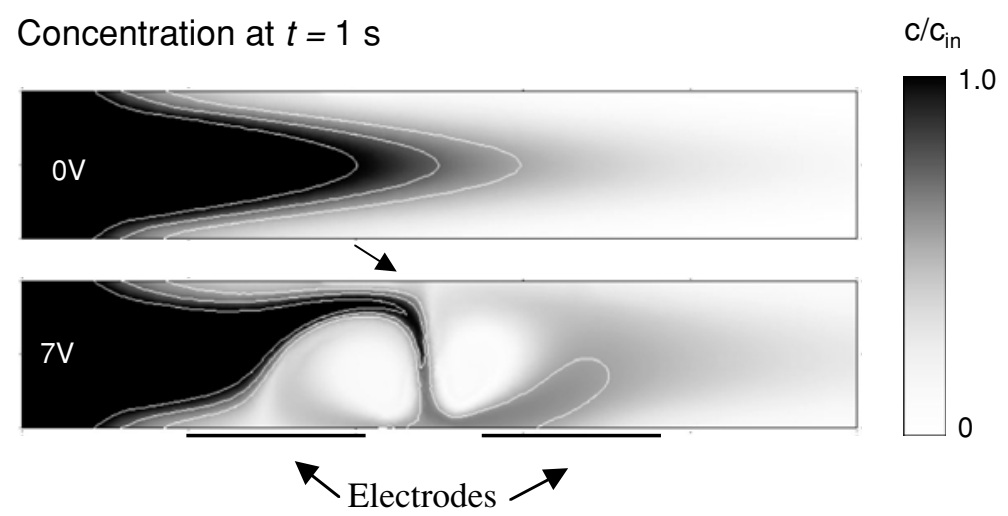

FIGURE 12.5. Concentration plots of electrothermally modified channel flow with applied voltages of $0 \mathrm{~V}$ and $7 \mathrm{~V}$. With optimal size and placement of electrodes, the electrothermal eddies can be engineered to span width of the channel, as is the case here, for a 40 micron channel. High concentration gradients and therefore an increase in diffusive flux in the vertical direction near the top channel wall indicate a favorable alternative location for the sensor here. 
to the electrodes, the electrothermally-induced motion transports the analyte close to the upper surface of the channel (Fig. 12.5b). This suggests that for these flow conditions and electrode configurations, an excellent sensor location is opposite the electrode gap.

Assuming a $1^{\text {st }}$ order heterogeneous reaction, the rate of binding is $k_{o n} C_{w}\left(R_{T}-B\right)$, where $k_{o n}=1 e 8 M^{-1} s^{-1}$ is the on-rate constant. The quantity, $R_{T}-B$, is the available antibody concentration, and $C_{w}(x)$ is the suspended concentration of antigen along the wall [14]. The off-rate is $k_{\text {off }} B$, where $k_{\text {off }}=.02 s^{-1}$ is the off-rate constant, and $B$ is the concentration of bound antigen. The time rate of change of antigen bound to the immobilized antibodies is equal to the rate of association minus the rate of dissociation

$$
\frac{\partial B}{\partial t} k_{o n} C_{w}\left(R_{T}-B\right)-K_{o f f} B .
$$

The rate of antigen binding to immobilized antigen, $\partial B / \partial$, must be balanced by the diffusive flux of antigen at the binding surface, $y=0$, such that

$$
\frac{\partial B}{\partial t}=\left.D \frac{\partial C}{\partial Y}\right|_{y=0} .
$$

Equations (12.21), (12.22) \& (12.23) are solved with an immobilized antibody concentration $R_{T}=1.7 \mathrm{nM} \mathrm{cm}$ (i.e. one molecule per $100 \mathrm{~nm}^{2}$ ). The binding rates for three conditions, 0, 7 and $14 \mathrm{Vrms}$, are shown in Fig. 12.6. The $0 \mathrm{Vrms}$ case corresponds to the passive case, which is the result of pure diffusion. This is the standard mode of most immobilized assays. The 7 and $14 \mathrm{Vrms}$ curves correspond to the result of electrothermally-driven flow enhancing the transport of antigen to the immobilized antibodies. The curves in

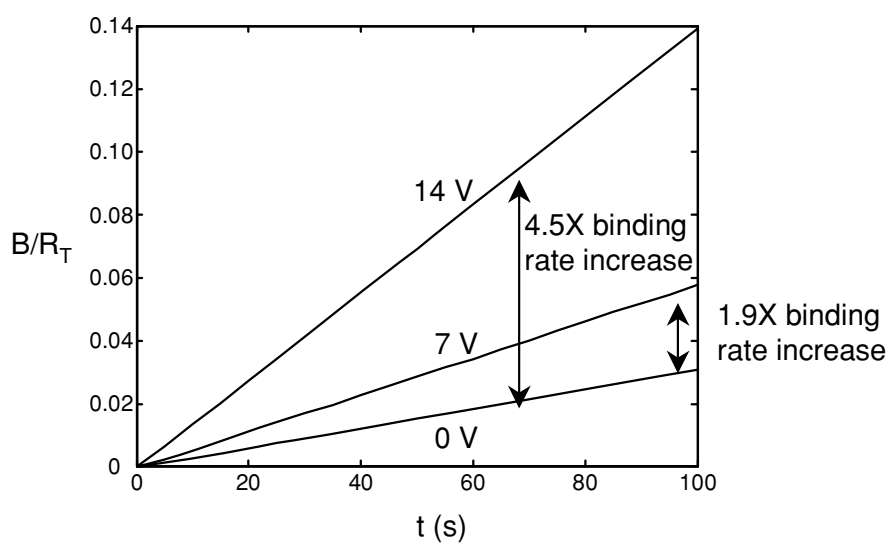

FIGURE 12.6. Numerical simulation of normalized bound concentration for a microchannel assay. The binding rate is increased by a factor of 2, when $7 \mathrm{Vrms}$ is applied to the electrodes. The binding rate is increased by a factor of 4.5, when $14 \mathrm{Vrms}$ is applied to the electrodes. These results suggest that electrothermally induced flow can significantly improve immunoassay performance by increasing binding rates. Parameters: Diffusivity, $D=$ $2 \times 10^{-11} \mathrm{~m}^{2} \mathrm{~s}^{-1}$ (corresponding to $20 \mathrm{~nm}$ spherical particle); inlet velocity is parabolic with average $100 \mu \mathrm{m} \mathrm{s}^{-1}$; inlet concentration $c_{0}=0.1 \mathrm{nM} ; \sigma_{w}=.00575 \mathrm{Sm}^{-1} ; k_{\text {on }}=1 e 8 \mathrm{M}^{-1} \mathrm{~s}^{-1} ; k_{\text {off }}=.02 \mathrm{~s}^{-1} ; R_{t}=1.67 e-11 \mathrm{Mm}$. 

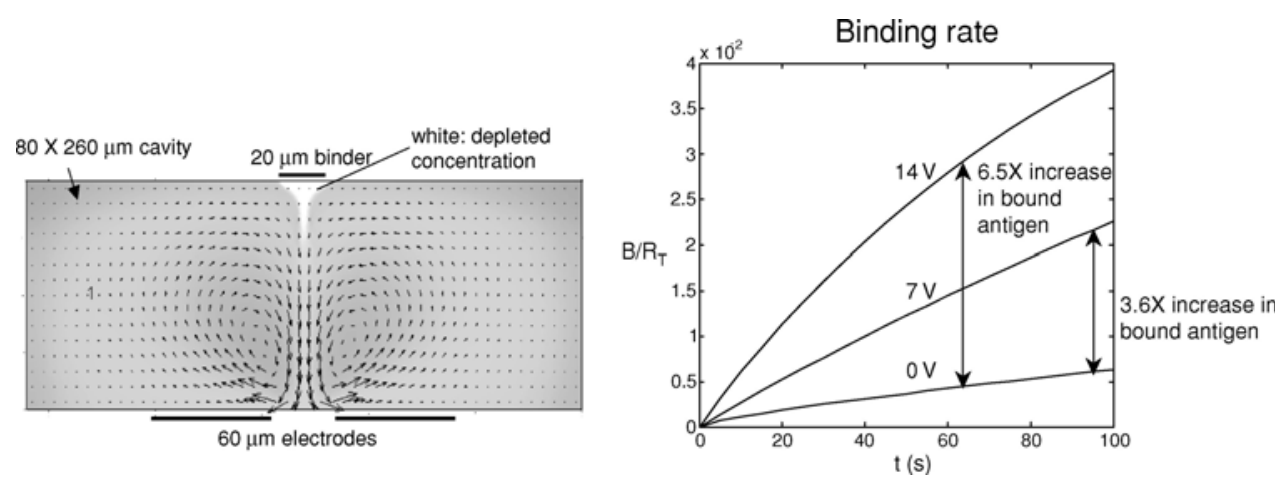

FIGURE 12.7. Microcavity (i.e. no flow-through) simulations (a) Velocity and concentration fields. The binder is centered above the electrodes; depleted concentration (white) is drawn down into the cavity. (b) Binding curves for non-enhanced $(0 \mathrm{~V})$ and enhanced $(7 \mathrm{~V}, 14 \mathrm{~V})$ transport. The differences in the two curves show an increase in binding rate which yields a factor of 3.6 higher binding for $7 \mathrm{~V}$ and a factor of 6.5 higher binding after 60 seconds for $14 \mathrm{~V}$ applied root-mean square potential. Paramters: Diffusivity, $10^{-11} \mathrm{~m}^{2} \mathrm{~s}^{-1}$; zero net flow; all other parameters identical to those in Fig. 12.3. Initial condition: $C(x, y)=C_{0}$ at $t=0$.

Fig. 12.6 show that a factor of 4.5 improvement in binding rate is obtained using ac electrokinetics in combination with a flowing microchannel.

Electrothermally-driven stirring can be used to improve assays for ELISA tests, microarray assays, and microtitre plates, where there is zero net flow. In these assays, the sample is often pre-mixed with a fluorescent or a chemiluminescent reporter, which increases the effective size of the analyte thereby decreasing its diffusivity. We simulate this effect by reducing the diffusivity by a factor of two, such that $D=10^{-11} \mathrm{~m}^{2} \mathrm{~s}^{-1}$.

The numerical simulation results are shown in Figure 12.7. The recirculating velocity field, which is characteristic of electrothermally-driven flow, is shown in Fig. 12.7a. This corresponds approximately to the streamlines shown in Fig. 12.2. For an applied voltage of 7 and $14 \mathrm{Vrms}$, the binding rate is increased by a factor of 3.6 and 6.5, respectively (see Fig. 12.7b). In the current simulation, the ac frequency is $f=100 \mathrm{kHz}$. In this range, the electrothermal velocity is not sensitive to changes in electrical frequency. At much lower frequencies $(\sim 100 \mathrm{~Hz})$, ac electroosmosis and electrode polarization typically dominate. At much higher frequencies $(\sim \mathrm{MHz})$, the dielectric component of the electrothermal force (last term in Eq. 12.18) dominates [17].

\subsection{CONCLUSIONS}

An analytical theory is presented that suggests that the combination of positive DEP and electrothermal fluid motion can produce non-localize trapping zones away from electrodes. The results suggest that this phenomenon could be used to focus bio-molecules in microfluidic sensors, when molecular diffusivity is low.

Numerical simulations are used to show how electrothermally-generated forces can be used to stir fluids in microchannels. Fluid velocities of approximately $\sim 400 \mu \mathrm{m} / \mathrm{s}$ are generated by applying potentials of $\mathrm{V}=14 \mathrm{Vrms}$ at $f=100 \mathrm{kHz}$. Precision stirring can 
be used to enhance the transport molecules towards functionalized surfaces. The results indicate that the binding rates of heterogeneous diffusion-limited reactions can be improved by a factor of 2-6, by applying 7-14 Vrms electrical potentials.

\section{ACKNOWLEDGMENTS}

This work has been supported by DARPA/ARMY DAAD19-00-1-0400, DARPA/Air Force F30602-00-2-0609, NSF CTS-9874839 and NSF ACI-0086061, and through the Institute for Collaborative Biotechnologies through grant DAAD19-03-D-0004 from the U.S. Army Research Office.

\section{REFERENCES}

[1] L. Bousse, C. Cohen, T. Nikiforov, A. Chow, A.R. Kopf-Sill, R. Dubrow, and J.W. Parce. Electrokinetically controlled microfluidic analysis systems [Review]. Ann. Rev. Biophys. Biomol. Struct., 29:155-181, 2000.

[2] D.-E. Chang, S. Loire, and I. Mezic. Closed-Form Solutions in the Electrical Field Analysis for Dielectrophoretic and Travelling Wave Inter-Digitated Electrode Arrays. Proceedings of the Conference on Decision and Control. Maui, HI, 2003.

[3] C.H. Chen and J.G. Santiago. A planar electroosmotic micropump. J. MEMS., 11(6):672-683, 2002.

[4] L.X. Chen, J.P. Ma, F. Tan, and Y.F. Guan. Generating high-pressure sub-microliter flow rate in packed microchannel by electroosmotic force: potential application in microfluidic systems. Sens. Actu. B-Chem., 88(3):260-265, 2003.

[5] C. Ring-Ling et al. Simultaneous hydrodynamic and electrokinetic flowcontrol. Micro Total Analysis Systems 2002, 1:386-388, Nov. 2002.

[6] V.V. Daniel. Dielectric relaxation. Academic Press, New York, 1967.

[7] P.R.C. Gascoyne and J. Vykoukal. Particle separation by dielectrophoresis [Review]. Electrophoresis, 23(13):1973-1983, 2002.

[8] N.G. Green, A. Ramos, and H. Morgan. AC electrokinetics: a survey of sub-micrometre particle dynamics, J. Phys. D, 33:632-641, 2000.

[9] M.P. Hughes. Electrophoresis, 23(16):2569, 2002.

[10] T.B. Jones. Electromechanics of Particles, Cambridge University Press, 1995.

[11] C.D. Meinhart, D. Wang, and K. Turner. Measurement of AC electrokinetic flows. J. Biomed. Microdev., 5(2):139-145, 2003.

[12] R. Miles, P. Belgrader, K. Bettencourt, J. Hamilton, and S. Nasarabadi. Dielectrophoretic manipulation of particles for use in microfluidic devices, MEMS-Vol. 1, Microelectromechanical Systems (MEMS). Proceedings of the ASME International Mechanical Engineering Congress and Exposition, Nashville, TN, Nov. 14-19, 1999.

[13] T. Muller, A. Gerardino, T. Schnelle, S.G. Shirley, F. Bordoni, G. DeGasperis, R. Leoni, and G. Fuhr. Trapping of micrometre and sub-micrometre particles by high-frequency electric fields and hydrodynamic forces. J. Phys. D.: Appl. Phys., 29, 340-349, 1996.

[14] D.G. Myszka. Survey of the 1998 optical biosensor literature. J. Mol. Recognit., 12:390-408, 1998.

[15] R. Pethig, Y. Huang, X.B. Wang, and J.P.H. Burt. Positive and negative dielectrophoretic collection of colloidal particles using interdigitated castellated microelectrodes. J. Phys. D.: App. Phys., 25:881-888, 1992.

[16] H.A. PohL. Dielectrophoresis. Cambridge University Press, 1978.

[17] A. Ramos, A. Castellanos, A. Gonzales, H. Morgan, and N. Green. Manipulation of Bio-Particles in Microelectrode Structures by means of Non-Uniform AC Electric Fields. Proceedings of ASME International Mechanical Engineering Conngress \& Exposition. New Orleans, LA Nov. 17-22, 2002.

[18] A. Ramos, H. Morgan, N.G. Green, and A. Castellanos. AC electrokinetics: a review of forces in microelectrode structures. J. Phys. D: Appl. Phys., 31:2338-2353, 1998. 
[19] W. Thormann, I. Lurie, B. McCord, U. Mareti, B. Cenni, and N. Malik. Advances of capillary electrophoresis in clinical and forensic analysis (1999-2000). Electrophoresis, 22:4216-4243, 2001.

[20] I. Tuval, I. Mezic, and O. Piro. Control of particles in micro-electrode devices. UCSB preprint 2004.

[21] X-B. Wang, J. Vykoukal, F. Becker, and P. Gascoyne. Separation of polystyrene microbeads using dielectrophoretic/gravitational field-flow-fractionation. Biophys. J., 74:2689-2701, 1998.

[22] D. Wang, M. Sigurdson, and C. Meinhart. Experimental analysis of particle and fluid motion in AC electrokinetics. Accepted in Exp. in Fluids. 2004.

[23] M. Washizu, O. Kurosawa, I. Arai, S. Suzuki, and N. Shimamoto. Applications of electrostatic stretch and positioning of DNA. IEEE Trans. Ind. Appl., 32(3):447-445, 1995.

[24] J. Yang, Y. Huang, X. Wang, X.-B. Wang, F. Becker, and P. Gascoyne. Dielectric properties of human leukocyte subpopulations determined by electrorotation as a cell separation criterion. Biophys. J., 76:33073314, 1999. 